\title{
La conception des transports collectifs en site propre (TCSP) en France : des tracés problématiques?
}

Pierre Zembri

\section{(2) OpenEdition}

Journals

Édition électronique

URL : http://journals.openedition.org/rge/3603

DOI : $10.4000 /$ rge.3603

ISSN : 2108-6478

Éditeur

Association des géographes de l'Est

Édition imprimée

Date de publication : 15 juin 2012

ISSN : 0035-3213

Référence électronique

Pierre Zembri, «La conception des transports collectifs en site propre (TCSP) en France : des tracés problématiques ? », Revue Géographique de l'Est [En ligne], vol. 52 / 1-2 | 2012, mis en ligne le 04 février 2013, consulté le 08 septembre 2020. URL : http://journals.openedition.org/rge/3603 ; DOI : https:// doi.org/10.4000/rge.3603

Ce document a été généré automatiquement le 8 septembre 2020

Tous droits réservés 


\title{
La conception des transports collectifs en site propre (TCSP) en France : des tracés problématiques?
}

\author{
Pierre Zembri
}

\section{Introduction}

1 Le tramway a été réintroduit dans les villes françaises au début des années 1980, après une disparition presque complète. Ne subsistaient des 101 réseaux initialement développés au 19ème siècle que trois lignes (Offner \& Zembri, 1994) à Marseille, SaintÉtienne et Lille. Le renouveau du tramway en France s'est traduit par une conception radicalement différente par rapport à la génération précédente :

- une séparation quasi-totale vis-à-vis de la circulation générale,

- des lignes plus longues (jusqu'à $20 \mathrm{~km}$ ) traversant les centres de part en part et se prolongeant dans des quartiers périphériques parfois éloignés,

- un souci de construction d'une image valorisante, à base d'un design innovant du matériel roulant, d'un mobilier urbain original et du choix de revêtements de sol parfois coûteux,

- un couplage urbanisme-transport se traduisant par un remodelage en profondeur des circulations, une requalification des quartiers traversés ainsi que par des aménagements de façade à façade, nettement plus coûteux que la simple pose des rails (Laisney, 2011).

Dans certains cas, les tracés des lignes ont été orientés par des programmes de financement public, notamment pour l'ouverture vers l'extérieur des quartiers défavorisés (politique dite de la ville). Dans d'autres cas, on a profité de la création du réseau pour traverser des zones futures d'urbanisation, ce qui donnait l'impression d'une inutilité complète du détour occasionné.

Au final, le tramway français de nouvelle génération donne l'impression d'un produit luxueux (pour les finances publiques), mais peu performant du fait des détours occasionnés par les opportunités de valorisation de quartiers ou par le souci de desservir un maximum de générateurs ou attracteurs de trafic. Il a été l'enjeu majeur 
de campagnes électorales, a occasionné la défaite de maires sortants comme celui d'Orléans en 2001, et continue à faire l'objet de débats passionnés au sein desquels des universitaires ont pu intervenir à charge (Carmona, 2001).

4 Si l'on se positionne sur le plan technique, il est vrai que les gains de temps par rapport à l'ancien système d'autobus sont souvent faibles, et ce pour au moins deux raisons :

- les tracés sont tortueux, avec de nombreux changements de direction. La ligne droite n'a pas été privilégiée et les distances se sont donc allongées par rapport aux lignes d'autobus, ce qui contrebalance les gains de vitesse éventuels du tramway,

- les vitesses prescrites pour les tramways sont plutôt faibles, parfois inférieures à celles des autobus à tracé comparable. C'est semble-t-il une question de confort des voyageurs. Elles peuvent être affectées par un degré de priorité insuffisant aux carrefours à feux.

La littérature scientifique est très rare sur cette question de la performance des tramways, qui semble constituer un « angle mort » de la recherche en transports. Les auteurs prennent généralement pour acquis le fait que les vitesses commerciales sont satisfaisantes, et suffisantes pour générer du report modal (Stambouli, 2007). Des risques potentiels de dégradation de ces vitesses ont cependant été recensés par JeanLouis Maupu (2003). Nous avons voulu approfondir ceux liés aux tracés et à la fixation des vitesses-limites. Nous avons pu travailler en première approche sur neuf réseaux : Nancy, Caen, Clermont-Ferrand, Bordeaux, Reims, Montpellier, Marseille, Toulouse et Orléans.

\section{Des itinéraires généralement originaux}

6 Les réseaux d'autobus des villes françaises sont les héritiers de réseaux de tramways créés entre 1855 et 1911, qui ont atteint leur développement maximal au début des années 1920 et qui ont été démantelés des années 1920 aux années 1970 (Robert, 1974). Le remplacement des lignes de tramway par des lignes d'autobus s'est effectué terme à terme, ce qui fait que l'on peut constater une grande permanence des itinéraires jusqu'à la veille de l'introduction du tramway « moderne ». Ce dernier est bien souvent l'occasion de refondre totalement le réseau alors que les transformations antérieures avaient le plus souvent consisté en des ajouts ou des prolongements de lignes au fur et à mesure des extensions de l'urbanisation ou du périmètre desservi (périmètre des transports urbains ou PTU).

7 L'introduction du tramway a en outre généré une hiérarchisation des lignes. Les nouvelles lignes « lourdes » sont protégées de la circulation générale, elles bénéficient d'une vitesse commerciale accrue et d'une plus grande régularité des circulations, et elles sont de surcroît celles où les capacités offertes sont les plus importantes. Les rames de tramway acheminent en effet entre 170 et 300 voyageurs contre 90 à 140 voyageurs pour les autobus. La desserte tramway est plus fréquente que celle des autobus. D'où la tentation de rabattre des lignes d'autobus sur les lignes tramways, en différents points, et de ne pas faire coexister sur le même axe les deux techniques.

8 Il est donc intéressant de comparer les nouveaux réseaux avec ceux qu'ils ont remplacés. Nous montrerons que les ruptures consistent essentiellement en l'adoption d'itinéraires moins évidents et moins droits qu'auparavant, ce qui peut s'expliquer par des objectifs de desserte modifiés, eux-mêmes parfois subordonnés à des objectifs de développement ou de re-développement urbains. 


\section{A. Des tramways... aux tramways en passant par l'autobus}

9 Les premiers réseaux de tramways français suivaient des axes principaux, et n'effectuaient que peu de détours. À titre d'exemple, la ligne A du tramway de Tours (situation de 1930) est absolument rectiligne entre ses terminus de la place de la Tranchée au Nord et de la Barrière de Grammont au Sud $(4 \mathrm{~km})$. Les lignes se croisaient en un ou plusieurs points dans les quartiers centraux : la place du Martroi à Orléans par exemple, la place Jean Jaurès et la place des Halles à Tours. Lorsque la configuration des centres urbains anciens (rues trop étroites ou trop tortueuses) interdisait la pénétration des lignes de tramways, celles-ci formaient une boucle comme cela fut le cas à Clermont-Ferrand. Mais elles s'éloignaient relativement peu des quartiers centraux denses, ce qui tranche singulièrement avec les réalisations actuelles.

Lorsque les réseaux de tramway originels ont été convertis à l'autobus, ce dernier n'a fait que reprendre les tracés existants, ce que montre très bien par exemple le travail récent réalisé sur Le Mans par Adelaïde de Ketelaere (2010). Par la suite, l'expansion considérable des agglomérations intervenue à partir des années 1960 a conduit dans un premier temps à allonger les lignes existantes, puis dans un second temps à créer des lignes permettant d'assurer une meilleure desserte des périphéries. Les axes antérieurement suivis par les tramways devenaient des troncs communs à plusieurs lignes d'autobus organisées en faisceaux.

11 Lors de la réintroduction du tramway «moderne » au début des années 1980, les agglomérations n'étaient plus aussi compactes que trente ans plus tôt. Par ailleurs, des pôles attractifs avaient été mis en place en périphérie: nouveaux campus universitaires, hôpitaux, zones d'activités, centres commerciaux et de loisirs. Des quartiers résidentiels d'habitat collectif dense avaient été également construits. Il était donc nécessaire de tenir compte de ces nouvelles polarités qui étaient parfois séparées du reste de l'agglomération par des espaces encore vides. D'où des lignes beaucoup plus longues, mais qui auraient pu être aussi rectilignes que possible.

\section{B. Le poids de la politique de revalorisation des quartiers défavorisés}

12 Rares sont les premières lignes des nouveaux réseaux qui ne desservent pas des zones d'habitat collectif social jugé défavorisé. Bellevue à Nantes, La Paillade à Montpellier, Hautepierre à Strasbourg, La Source à Orléans, Les Caillols à Marseille, Croix-Rouge et Orgeval à Reims bénéficient dès le départ d'une desserte en tramway. Ces choix peuvent se justifier par un taux de motorisation des habitants moins élevé que la moyenne, et donc des difficultés d'accès à des emplois généralement éloignés. En effet, l'évolution socio-économique de tels quartiers est très défavorable depuis le début des années 1980 du fait du départ des populations les plus solvables, encouragées par les aides d'État à accéder à la propriété. Ces dernières ont été remplacées par des ménages plus précaires, logés là où il $\mathrm{y}$ avait des logements disponibles par les bailleurs sociaux propriétaires de la plus grande partie de ces quartiers. D'où le lancement de politiques destinées à empêcher la fermeture progressive de ces quartiers et à permettre à leurs habitants actifs de se déplacer dans de meilleures conditions pour accéder aux emplois. On les regroupe sous le vocable général de politiques de la Ville. 
13 Les politiques de la Ville ont été mises en place à partir de 1984. Le développement de transports publics performants desservant les quartiers « sensibles » a été rapidement érigé au rang de priorité, de façon à les ouvrir vers l'extérieur. Des financements ont été proposés aux gestionnaires d'agglomération pour qu'ils orientent leurs lignes de tramway vers les quartiers bénéficiant d'un label Politique de la ville ${ }^{1}$. À la mise en relation s'ajoute la rénovation des espaces publics traversés par les nouvelles infrastructures et l'embellissement global des quartiers, ce qui contribue à la revalorisation de leur image de marque ainsi qu'à l'attraction d'activités ou de services.

14 Le complément de financement promis a profité à la plupart des agglomérations développant un TCSP. Ainsi, l'agglomération de Clermont-Ferrand bénéficie actuellement de 30 millions d' $€$ de la part de l'État pour prolonger sa ligne A de 1,7 km au-delà de son terminus septentrional actuel, de façon à desservir les quartiers de Champratel et des Vergnes.

\section{Passer partout ? Les risques d'une volonté de desserte trop exhaustive}

15 L'impression que peut ressentir un observateur non averti est que les concepteurs des premières lignes construites dans une agglomération donnée ont considéré qu'elles demeureraient des exemplaires uniques, et qu'il convenait de leur faire desservir l'ensemble des générateurs et attracteurs de trafics dans de vastes secteurs. On constate objectivement que les lignes n'adoptent pas comme leurs devancières des tracés droits suivant de façon continue les grands axes convergeant vers le centre d'agglomération. Le cas de Montpellier est très représentatif de cette approche combinant parcours radiaux ( $\mathrm{du}$ centre vers la périphérie) et parcours de rocade. La première ligne relie le centre commercial et de loisirs d'Odysseum (à l'est de l'agglomération) au quartier de La Paillade (à l'ouest) en passant par la gare et la Place de la Comédie, centre emblématique de la ville. Sur sa partie orientale, elle effectue un crochet pour desservir le nouveau quartier d'Antigone - Port Marianne, qui accueille notamment les sièges du Conseil régional et de la Communauté d'agglomération. Après un passage très tourmenté dans le quartier de la gare, elle contourne le centre ancien par l'Est et le Nord avant de gagner le quartier des universités au Nord-Ouest, puis des hôpitaux universitaires. Elle se détache ensuite des axes à dominante radiale pour effectuer un crochet vers le Sud-Est, ce qui lui permet d'entrer dans le quartier de la Paillade et de terminer au stade de la Mosson. Les équipements importants du quart nord-ouest de Montpellier sont donc desservis par une seule ligne alors qu'ils auraient été du ressort de trois lignes distinctes d'autobus suivant des tracés radiaux directs (figure 1). Nous verrons plus loin que ce n'est pas sans conséquence sur les temps de parcours de bout en bout. 
Figure 1 : Comparaison entre tracés directs sur voirie et tracés suivis par les deux lignes de TCSP de Montpellier.

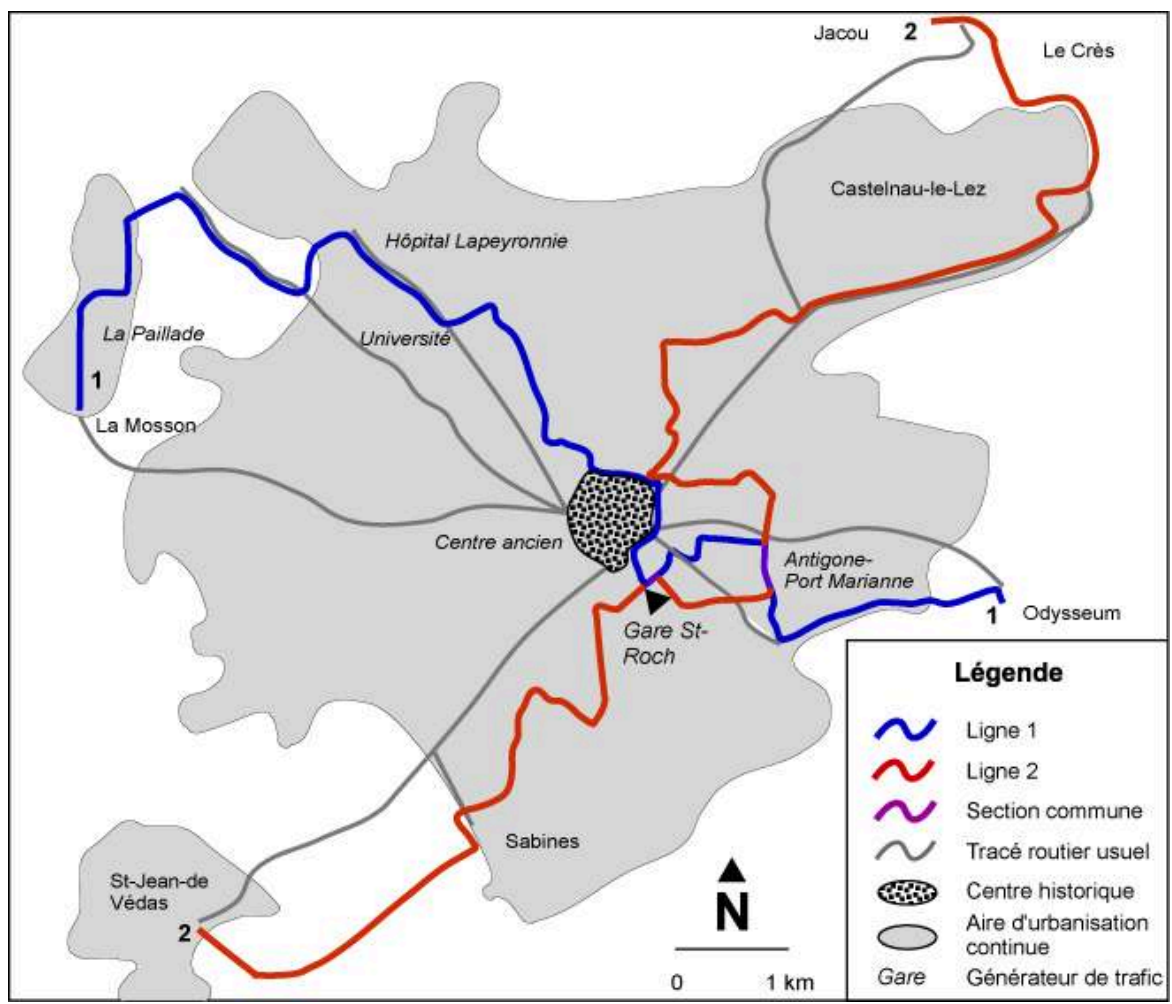

16 À l'objectif de desserte d'un maximum de pôles générateurs de trafic s'ajoute parfois celui de passer par des secteurs de développement futur de l'agglomération qui ne sont pas encore urbanisés au moment de la construction du TCSP. Cette option, qui avait été prise pour la première fois à Orléans en 2000 (Archambault, 1995), avait suscité des réactions très négatives : il est vrai que la ligne traverse des vergers et des terrains en friche sur près de deux kilomètres entre Olivet et l'Université d'Orléans (campus de La Source). Le détour occasionné par rapport à un itinéraire direct est important, ce qui a des conséquences non négligeables sur le temps de parcours. Par la suite, l'agglomération de Montpellier a pris également le parti pour sa seconde ligne de traverser des secteurs encore ruraux entre les Sabines et Saint-Jean de Védas (au SudOuest) et entre Castelnau-le-Lez et Jacou (au Nord-Est). Encore plus récemment, la première ligne TCSP de Reims, ouverte en avril 2011, comporte une branche à destination de la gare périphérique de Champagne-Ardenne TGV. Cette branche traverse sur plus de la moitié de sa longueur $(1,4 \mathrm{~km})$ une zone d'urbanisation future (ZAC de Bezannes), et deux emplacements ont été réservés pour de futures stations intermédiaires. La fréquentation de cette branche repose aujourd'hui en grande partie sur la clientèle de/vers la gare TGV, qui peut par ailleurs prendre des trains régionaux plus rapides vers le centre. Elle risque donc de rester très faible jusqu'à l'urbanisation effective de ce secteur. 
Figure 2 : Comparaison entre tracés directs sur voirie et tracés suivis par la ligne A du TCSP d'Orléans

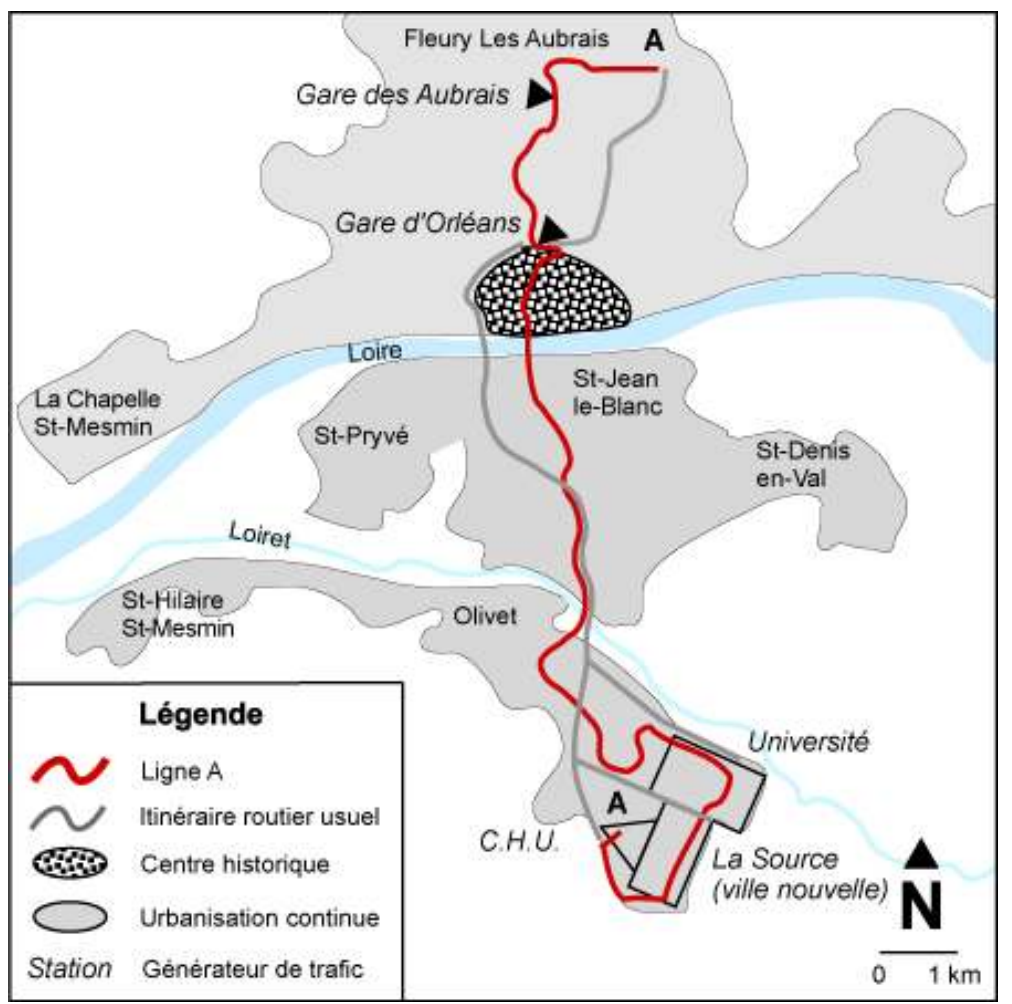

Il arrive également que l'on fasse au TCSP le procès inverse lorsqu'il ne dessert pas un équipement d'agglomération jugé fondamental comme la gare centrale. C'est ce qui s'est produit à Clermont-Ferrand. La première ligne de TCSP, d'orientation générale, Nord-Sud, contourne le centre ancien par l'Ouest alors que la gare est à l'Est. On notera que l'ancien réseau en faisait de même, et que la gare n'était desservie que par un embranchement en cul-de-sac (Robert, 1974). L'opposition au tracé proposé a été telle que l'affaire est remontée jusqu'au Conseil d'État, instance supérieure de la justice administrative française, et ce dernier a donné raison à la communauté d'agglomération, aucun texte n'obligeant à desservir une gare !

\section{Des tracés tourmentés, des limitations de vitesse nombreuses}

Notre analyse va désormais se focaliser sur les tracés eux-mêmes : ils se caractérisent, du fait de la multiplicité des missions confiées au TCSP, par de nombreux changements de direction et par l'emprunt de voiries secondaires, moins larges et moins droites que les voies principales habituellement suivies. Mais on trouve également des tracés indépendants de la voirie en périphérie qui font passer le tramway pour un véritable chemin de fer suburbain, et qui permettent de faire remonter les vitesses moyennes calculées sur l'ensemble des lignes. Cela dit, ceux qui vont le plus vite aux extrémités des lignes ne sont pas forcément les plus nombreux... 


\section{A. Des changements de direction fréquents}

19 Les changements d'axe dans les périphéries et les parcours dans les quartiers centraux se traduisent par des courbes de faible rayon, qui occasionnent des ralentissements par rapport aux vitesses pratiquées en ligne droite. Mais d'autres facteurs peuvent aussi jouer comme la sinuosité des voies suivies par le TCSP, le nombre de traversées routières du site réservé, l'ouverture de la plate-forme du TCSP aux piétons (dans les centres anciens), etc. Dans le cas de Reims, un principe de précaution que le maître d'ouvrage s'est auto-imposé (aucun réseau n'avait procédé de la sorte auparavant) veut que les conducteurs diminuent leur vitesse de $10 \mathrm{~km} / \mathrm{h}$ à toutes les intersections, même en ligne droite! Nous verrons plus loin que cela se traduit par une multitude de changements de vitesse-limite, impossibles à respecter par les conducteurs.

D’une façon générale, les vitesses pratiquées en bout de ligne dans les périphéries sont largement supérieures à celles prescrites dans les quartiers centraux. La plupart des tramways récents sont autorisés à circuler à $70 \mathrm{~km} / \mathrm{h}$, surtout lorsque les lignes traversent des zones de faible densité. En revanche, les parcours en zone dense peuvent donner lieu à des restrictions sévères : $20 \mathrm{~km} / \mathrm{h}$ en général dans les zones piétonnes, 30 à $50 \mathrm{~km} / \mathrm{h}$ le long des voiries. L'accumulation des taux réduits a un impact sur les temps de parcours non négligeable.

21 Les changements de direction sont nombreux, même si leur fréquence n'est pas la même d'un réseau à l'autre. Dans le cas de Reims, on en relève un tous les 658 mètres en moyenne, dont dix avec un angle supérieur à $45^{\circ}$. Sur la ligne A d'Orléans, on compte 24 courbes sur les $18 \mathrm{~km}$ du parcours, soit une tous les $750 \mathrm{~m}$ en moyenne. Sur la ligne $2 \mathrm{de}$ Montpellier, 24 changements de direction sur 19,8 km. Si les tramways classiques admettent un rayon de giration minimum de 20 mètres, les tramways sur pneus développés à Nancy, Caen et Clermont-Ferrand peuvent franchir des courbes de plus faible rayon : 10,5 m pour le Translohr et $12 \mathrm{~m}$ pour le TVR (données fournies par les constructeurs). Les concepteurs des réseaux n'ont pas hésité à leur imposer des courbes en rapport avec leurs performances supposées. Dans le cas de Nancy, le TVR a tendance à se désolidariser de son rail du fait de la trop faible pression exercée par le dispositif de guidage: après plusieurs déraillements, il a fallu se résoudre à le limiter sur les courbes les plus accentuées à la vitesse dérisoire de $5 \mathrm{~km} / \mathrm{h}$. En poussant le système à ses limites, on en diminue fortement les performances réelles. Les tramways classiques passent également des courbes aux limites de leurs capacités, ce qui occasionne des ralentissements à 10 ou $15 \mathrm{~km} / \mathrm{h}$.

22 On peut admettre exceptionnellement sur un tracé une courbe de faible rayon liée à la configuration de la voirie. Il est en revanche difficilement acceptable de les multiplier dans la mesure où au temps perdu par les passagers s'ajoute une usure plus importante du matériel roulant trop régulièrement sollicité.

\section{B. Trois illustrations complémentaires : Orléans, Montpellier et Reims}

23 Ces trois réseaux sont proches par leurs caractéristiques: des lignes traversant des environnements urbains variés, des rues et places piétonnes de l'hypercentre aux terrains non encore urbanisés de périphéries parfois lointaines, en passant par des quartiers d'habitat collectif excentrés et par des centres commerciaux. Ils comptent ou 
sont en voie de compter chacun (dans le cas d'Orléans, la ligne B est actuellement en construction) deux lignes.

Dans les trois cas, les lignes sortent de la zone urbaine dense et traversent des territoires quasiment vides, avec moins d'arrêts et de bons profils. Dans les deux premiers cas, on traverse des zones d'urbanisation future que le passage du tramway est censé développer dans une logique d'urbanisme durable. Dans le cas de Montpellier, il s'agit d'aller desservir des périphéries éloignées (Saint-Jean de Védas, Jacou) en traversant des espaces non urbanisés qui n'ont pas forcément vocation à se développer. Les lignes sont de longueurs variées : celles de Reims sont plus courtes (respectivement $9,5 \mathrm{~km}$ et 6,9 km) avec un tronc commun de 3,5 km. Les deux lignes de Montpellier sont longues de 15,2 km (ligne 1) et de 19,8 km (ligne 2). La première ligne d'Orléans compte $18 \mathrm{~km}$.

On peut noter que dans les trois cas les tracés sont plutôt tourmentés, ce qui laisse penser que les vitesses les plus élevées (supérieures ou égales à $50 \mathrm{~km} / \mathrm{h}$ ) ne seront autorisées au plus que sur 40 à $60 \%$ de la longueur totale. En pratique (tableau 1), ce n'est le cas que sur $51 \%$ du tracé à Reims. Ce qui frappe dans cet exemple, c'est le grand nombre de taux de vitesses prescrits: dix au total, sur 101 tronçons! On ne retrouve heureusement pas de telles fréquences sur les deux autres réseaux.

Tableau 1 : Fréquence et longueur moyenne des zones de limitation de vitesse par taux sur les deux lignes du TCSP de Reims (sens Nord-Sud, état au 25 novembre 2010).

\begin{tabular}{|c|c|r|r|}
\hline $\begin{array}{c}\text { Taux applicable }(\mathrm{km} / \\
\mathrm{h})\end{array}$ & $\begin{array}{c}\text { Nombre de } \\
\text { zones }\end{array}$ & $\begin{array}{c}\text { Longueur cumulée } \\
(\mathrm{m})\end{array}$ & $\begin{array}{c}\text { Longueur moyenne } \\
(\mathrm{m})\end{array}$ \\
\hline 15 & 13 & 950 & 73 \\
\hline 20 & 5 & 820 & 164 \\
\hline 25 & 8 & 670 & 84 \\
\hline 30 & 9 & 610 & 68 \\
\hline 35 & 2 & 2160 & 140 \\
\hline 40 & 30 & 4360 & 155 \\
\hline 50 & 28 & 80 & 80 \\
\hline 55 & 1 & 600 & 150 \\
\hline 60 & 4 & 680 & 680 \\
\hline 70 & 1 & & 72 \\
\hline
\end{tabular}

Il est intéressant d'établir un parallèle entre les vitesses autorisées et le contexte urbain des lignes de tramway, en partant du principe que les contextes les plus denses sont ceux où la fréquentation sera la plus importante. Or, comme nous allons le voir, c'est aux extrémités des lignes, en milieu périurbain, que les vitesses pratiquées sont 
les plus élevées, ce qui permet de faire la moyenne avec les vitesses plus faibles des zones centrales.

La seconde ligne du TCSP de Montpellier (Saint-Jean de Védas - Jacou) constitue un exemple représentatif de cette configuration. Elle comporte en effet de longs tronçons en milieu semi-rural, parcourus à $70 \mathrm{~km} / \mathrm{h}$ avec des distances entre arrêts nettement plus longues. Ces tronçons ne sont parcourus que par une rame sur deux, des terminus intermédiaires ayant été ménagés aux Sabines et à Sablassou : les extrémités des lignes sont de ce fait à voie unique, des croisements étant possibles dans la plupart des stations. Entre les Sabines et l'entrée de Saint-Jean de Védas, la ligne 2 reprend le tracé d'une voie ferrée du réseau national fermée à tout trafic ferroviaire classique (photo 1). Entre Sablassou et le terminus Nord-Est de Jacou, le tracé est totalement indépendant de la voirie, mais il ne s'agit pas de la reprise d'une ancienne voie ferrée (photo 2).

Photo 1 : Vue de la ligne 2 du tramway de Montpellier sur le tronçon purement ferroviaire entre les Sabines et St-Jean de Védas (cliché : Pierre Zembri)

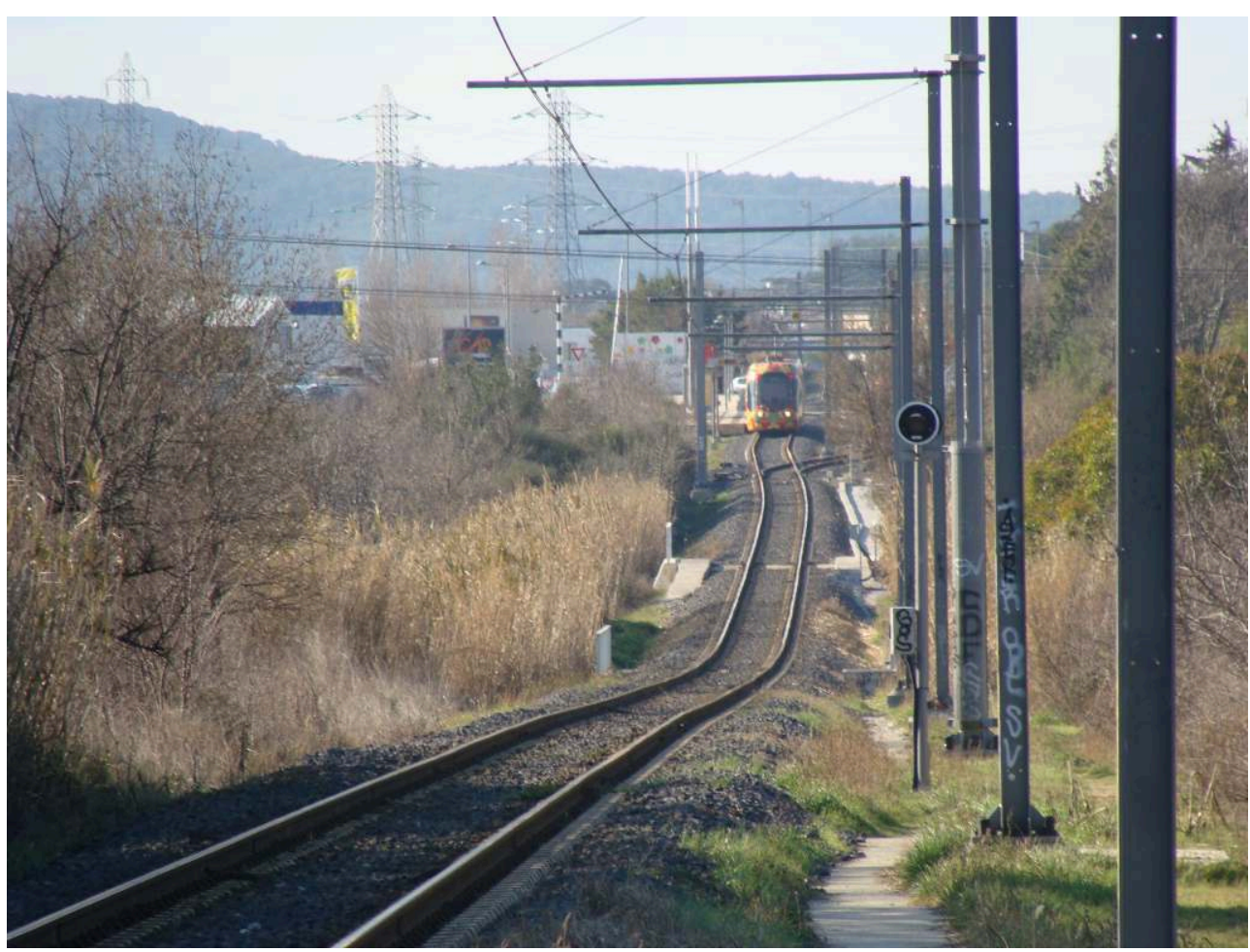


Photo 2 : Tracé indépendant de la ligne 2 du tramway de Montpellier entre Sablassou et Jacou (cliché Pierre Zembri)

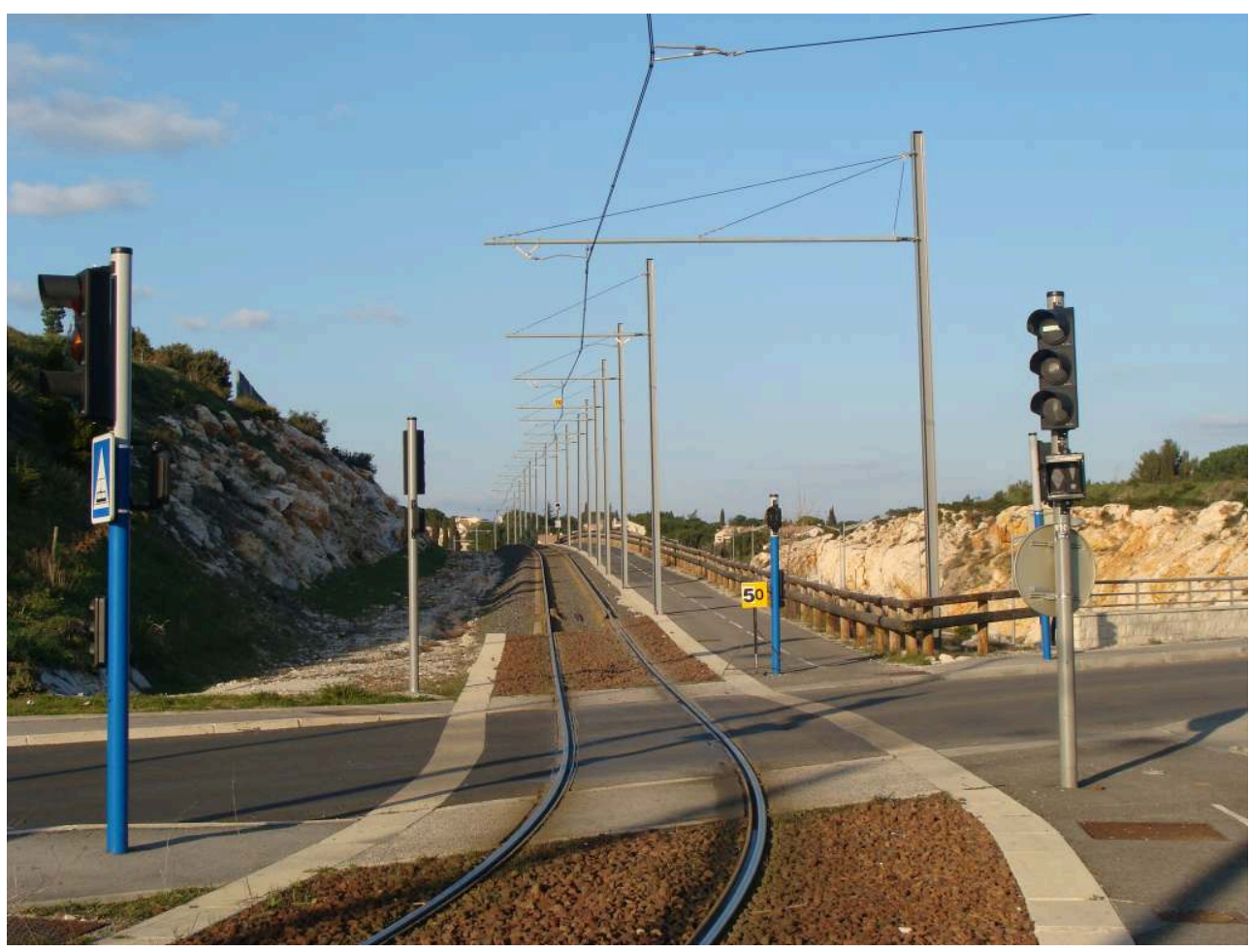

En revanche, dans le centre de l'agglomération, les vitesses sont plus faibles avec un parcours particulièrement sinueux aux abords du Corum et de la gare SNCF, où les vitesses sont réduites à $20 \mathrm{~km} / \mathrm{h}$, voire $10 \mathrm{~km} / \mathrm{h}$.

Le même type d'organisation peut être observé sur le réseau d'Orléans. La ligne relie le centre d'Orléans à la ville satellite de La Source, qui associe l'Université, des quartiers d'habitat collectif, des entreprises et un centre hospitalier d'importance régionale. Elle traverse entre les deux des secteurs encore ruraux où les vitesses pratiquées peuvent être plus élevées (photo 3), en suivant pour partie une voie routière rapide. En revanche, elle est loin d'adopter le parcours le plus direct, ce qui la rend comme nous le verrons plus loin peu performante par rapport à la desserte bus antérieure. 
Photo 3 : la ligne A du tramway d'Orléans entre Orléans et La Source, dans un secteur non encore urbanisé de la commune d'Olivet (cliché : Pierre Zembri)

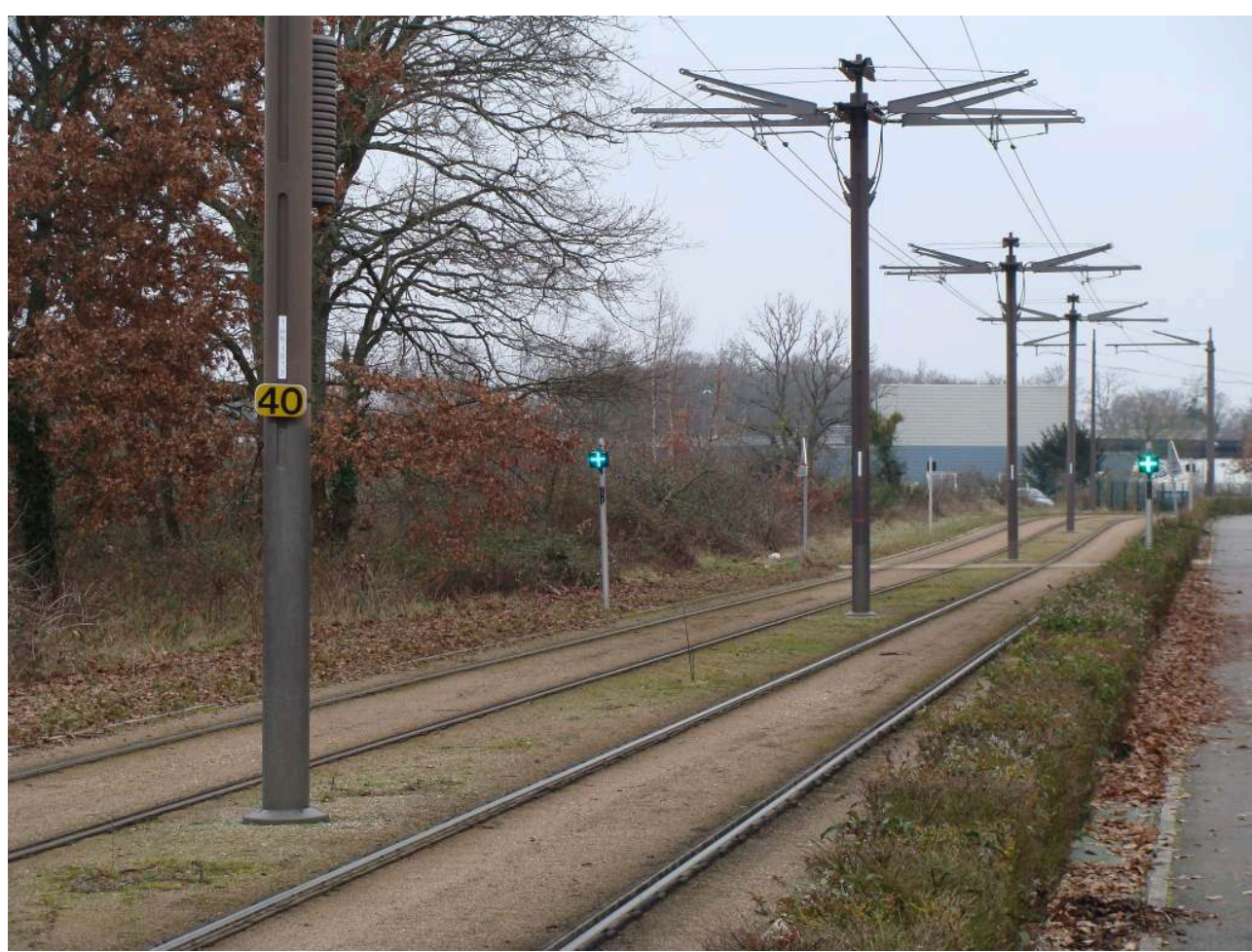

Dans le cas de Reims, la ligne B, qui relie la gare centrale à la gare TGV, traverse la future zone de développement urbain de Bezannes, avec un tracé à voie unique. C'est sur ce tronçon que les rames peuvent circuler à $70 \mathrm{~km} / \mathrm{h}$, mais avec peu de clients à acheminer. En revanche, les parties de ligne dans les quartiers centraux voient les tramways circuler à vitesse nettement plus réduite.

\section{Les défauts d'une approche trop globale des vitesses commerciales}

L'affichage d'une vitesse commerciale globale par ligne, voire pour l'ensemble des lignes de tramway d'une ville, est de nature à tromper décideurs et utilisateurs, tout en générant une forte pression sur les conducteurs pour que les temps de parcours soient respectés. C'est ce que nous allons démontrer, en nous appuyant sur quelques exemples sur lesquels nous avons pu avoir des informations détaillées.

\section{A. Définition et affichage}

La vitesse commerciale se définit comme la vitesse moyenne calculée de terminus à terminus, en tenant compte des arrêts, et des conséquences de tous les aléas rencontrés sur le parcours (congestion, défaut de réglage des feux tricolores, affluence exceptionnelle, etc.).

34 Les vitesses annoncées lors des projets sont assez homogènes : autour de $20 \mathrm{~km} / \mathrm{h}$. Les vitesses réelles après mise en service sont plus variées : entre $16,7 \mathrm{~km} / \mathrm{h}$ (Nantes ligne 3) et $22,2 \mathrm{~km} / \mathrm{h}$ (Nantes ligne 1) (Stambouli, 2007). Jacques Stambouli fait valoir que, 
même légèrement inférieures aux performances annoncées, ces vitesses sont tout à fait comparables à celles d'un parcours automobile équivalent ${ }^{2}$. Mais peut-on parler d'équivalence des parcours compte tenu des écarts par rapport à la ligne droite que nous avons mis en évidence dans la partie précédente?

En outre, la vitesse commerciale globale est trompeuse car elle peut masquer des disparités importantes, ce que nous allons voir par la suite. Ce n'est de surcroît pas un indicateur pertinent de la performance des lignes de TCSP dans la mesure où les passagers sont plutôt sensibles aux temps de parcours réels de point à point. Enfin, elle détermine la fixation des horaires et des tableaux de marche pour l'exploitant: l'objectif politique, même irréaliste, devient le seuil en deçà duquel des pénalités financières peuvent être appliquées, avec les conséquences que l'on imagine sur les conditions de travail des conducteurs.

\section{B. Vitesses calculées et temps de parcours ressenti}

Pour reprendre les termes de Jean-Louis Maupu (2003), «la ligne ne passe pas là où l'usager l'attend. (...) Une ligne sinueuse peut devenir un grand manège très bien rempli, mais est-ce l'objectif?». Il convient donc d'approfondir ce paradoxe, qui voit des performances réelles plutôt médiocres ne pas empêcher de bons niveaux de fréquentation.

Dans le cas de Montpellier, nous avons vu plus haut que les parcours du tramway sont sensiblement plus longs dans la mesure où ils combinent plusieurs liaisons qui étaient auparavant assurées par des lignes d'autobus distinctes, aux tracés plus directs. Nous avons tenté d'effectuer une comparaison entre les distances parcourues par le tramway et les distances par voie routière directe. Comme le montre le tableau 2, le tramway est toujours perdant, même si la circulation routière doit contourner le centre ancien.

Tableau 2 : Comparaison entre distances en tramway et sur tracés routiers directs à Montpellier

\begin{tabular}{|l|r|r|r|r|}
\hline & $\begin{array}{r}\text { Route } \\
(\mathrm{m})\end{array}$ & Tramway $(\mathrm{m})$ & $\begin{array}{r}\text { Différence } \\
(\mathrm{m})\end{array}$ & $\begin{array}{r}\text { Différence } \\
(\%)\end{array}$ \\
\hline Gare - St-Jean de Védas & 4900 & 7090 & 2190 & $44,69 \%$ \\
\hline Gare - Sabines & 3000 & 4080 & 1080 & $36,00 \%$ \\
\hline Gare - Odysseum & 3500 & 5870 & 2370 & $67,71 \%$ \\
\hline Gare - Sablassou & 6000 & 8700 & 2700 & $45,00 \%$ \\
\hline Gare - Jacou & 7500 & 12710 & 5210 & $69,47 \%$ \\
\hline Gare - Euromédecine & 5440 & 6790 & 1350 & $24,82 \%$ \\
\hline Gare - Mosson ${ }^{3}$ & 5070 & 9330 & 4260 & $84,02 \%$ \\
\hline Gare - Université & 3700 & 4350 & 650 & $17,57 \%$ \\
\hline
\end{tabular}

Calculs effectués à partir des fonds cartographiques de l'IGN 
Le parcours Gare - Jacou est effectué en 35 minutes en tramway ${ }^{4}$, tandis que les 7500 mètres du trajet routier demanderaient 26,5 minutes à $17 \mathrm{~km} / \mathrm{h}$ de vitesse moyenne, soit $24,3 \%$ de moins, ce qui est très important pour une agglomération de cette taille. Le parcours Gare - Saint-Jean de Védas est effectué en 21 minutes en tramway, contre 17,3 minutes par la route (17,6 \% de moins). Dans ces deux cas de figure, la vitesse élevée du tramway en périphérie lui permet de limiter le différentiel de temps par rapport à la route (la distance est respectivement plus longue de 69,47 et 44,69\%). Pour les parcours plus tourmentés comme Gare - Mosson, le tramway met presque deux fois plus de temps qu'un véhicule routier circulant à $17 \mathrm{~km} / \mathrm{h}$ (31 minutes contre 17,8).

Dans le cas d'Orléans, la situation est comparable pour les liaisons entre les quartiers centraux et le quartier de La Source, avec une mention spéciale pour la desserte de l'hôpital régional qui est desservi après un détour important par le campus universitaire. Par la route, il est à $11 \mathrm{~km}$ de la gare (soit un temps de parcours de 17 minutes ${ }^{5}$ ). Par le tramway, le temps de parcours est de 35 minutes. Le campus universitaire lui-même est relié à la gare, lieu d'arrivée de nombreux étudiants, en 28 minutes, temps peu différent de celui que mettaient les autobus avant la mise en service du tramway. Par la route, les 11 kilomètres sont parcourus en 20 minutes (soit $28,5 \%$ de moins).

Dans les deux cas cités, les vitesses élevées sur une partie du parcours compensent partiellement les surcroîts de distance, mais ne parviennent pas à les effacer. Il en résulte des performances insuffisantes de point à point, alors que les vitesses commerciales résultant des horaires officiels sont plutôt élevées $(21,6 \mathrm{~km} / \mathrm{h}$ pour la ligne A d'Orléans, $21,2 \mathrm{~km} / \mathrm{h}$ pour la ligne 2 de Montpellier). La fréquentation est logiquement plus importante sur les parties de lignes les plus directes.

\section{Vitesses imposées et travail de conduite}

41 En pratique, les vitesses commerciales annoncées au moment de la présentation des projets sont rarement celles mesurées une fois les lignes mises en service. La déperdition peut s'expliquer par un réglage déficient des priorités aux carrefours, mais aussi par des modifications en cours de projet des vitesses-limites fixées sur différents tronçons. Il revient aux conducteurs de se rapprocher autant que possible de la performance annoncée, quelles que soient les conditions de conduite et la fréquentation des rames.

Or, les aléas sont nombreux sur un parcours : véhicules routiers immobilisés sur le site du tramway, affluence de piétons dans les rues commerçantes des centres villes, feux tricolores en dérangement, etc. Par ailleurs, les horaires intègrent des temps d'arrêt en station qui ne peuvent pas toujours être respectés : les principaux arrêts donnant des correspondances multiples en centre d'agglomération peuvent donner lieu à des surstationnements importants en cas d'affluence. Ces derniers peuvent être aggravés par le positionnement des valideurs de titres de transport à l'intérieur des rames, au niveau des portes. Certains réseaux, comme celui de Strasbourg, ont choisi de positionner les valideurs sur les quais et non dans les rames, mais c'est loin d'être la règle ailleurs. Quelle que soit la nature des aléas rencontrés, il revient aux conducteurs de rattraper autant que possible les retards accumulés pour éviter aux exploitants des pénalités pour non-respect des horaires. Cela passe par des pauses écourtées aux terminus ou par des comportements de conduite non conformes au souci de confort des voyageurs. 
43 La pression est devenue telle sur certains réseaux que des conflits sociaux ont pu être générés par des problèmes de respect des horaires imposés. Ce fut notamment le cas sur la ligne T3 du réseau parisien, à la suite de la décision de la direction d'augmenter la vitesse commerciale sur la ligne sans que les conditions d'exploitation aient été modifiées.

\section{Conclusions}

Les performances des TCSP français sont donc paradoxales : des vitesses commerciales plutôt élevées, sur des parcours plus longs que ceux antérieurement suivis par les autobus. Au final, les temps de parcours de point à point (notamment des extrémités des lignes au centre ancien et à la gare) sont décevants. Les itinéraires dévient assez fortement de la ligne directe la plus évidente, et les détours dictés par le souci de desservir la proportion de populations et d'emplois la plus élevée possible nuisent à l'efficacité du système. Le remplissage des rames est donc très satisfaisant dans la partie centrale, là où les détours ont le moins d'impact négatif, mais beaucoup moins ailleurs.

Les détours occasionnés sont de surcroît source de contraintes pour le matériel roulant, souvent poussé aux limites de ce qu'il peut accomplir, notamment pour ce qui concerne les rayons de courbure. Les systèmes sur pneus (TVR, Translohr) sont ceux qui sont le plus sollicités. L'accumulation des courbes et des restrictions de vitesse associées multiplie les occasions de rouler au-dessous de la vitesse nominale, ce qui réduit la marge de manœuvre des conducteurs, tenus de respecter des horaires trop contraignants.

On peut comprendre que les décideurs locaux aient souhaité que les TCSP, beaux objets urbains, symboles de la modernité voire du renouveau urbain, soient visibles par le plus grand nombre dès la mise en service de la première ligne. Mais la dimension urbanistique l'a emporté sur la dimension transport, au détriment des performances en termes de vitesse. Si un réseau comme Montpellier a récemment évolué dans le sens d'un plus grand maillage, ce qui a permis d'échanger des tronçons entre lignes et de mettre en place des itinéraires plus directs, il n'est pas certain que ce mouvement se généralise.

\section{BIBLIOGRAPHIE}

Archambault M.-F., 1995, « Le tramway d'Orléans, vecteur d'urbanisme ? », Transports Urbains, 95, Avril - Juin, pp. 9-14.

Carmona M., 2001, Tramway, le coût d'une mode, Orléans, Editions Paradigme, 200 p.

De Ketelaere A., 2010, La politique des transports urbains au Mans (1947-1990), Mémoire de Master d'Histoire, Université de Mans.

Revue Géographique de l'Est, vol. 52 / 1-2 | 2012 
Laisney F., 2011, Atlas du tramway dans les villes françaises, Paris, Editions Recherches, 422 p.

Maupu J.-L., 2003, « Comment choisir un système de transport collectif en site propre (TCSP) de surface? », Transports Urbains, 103, Octobre - Décembre, pp. 11-19.

Offner J.-M., Zembri P., 1994, « Tramway, transport public : histoires parallèles », Transport Public, Janvier, pp. 20-29.

Orfeuil J.-P., 2000, L'évolution de la mobilité quotidienne, comprendre les dynamiques, éclairer les controverses, Synthèse INRETS $n^{\circ} 37$, Novembre.

Robert J., 1974, Histoire des transports dans les villes de France, Neuilly-sur-Seine, 539 p.

Stambouli J., 2007, «Les territoires du tramway moderne : de la ligne à la ville durable ", Développement durable et territoires, Dossier $4:$ La ville et l'enjeu du Développement Durable, URL : http://developpementdurable.revues.org/3579

\section{NOTES}

1. Il y a eu plusieurs classements successifs ou simultanés : Zones urbaines sensibles (ZUS), Zones franches urbaines (ZFU), quartiers prioritaires des contrats urbains de cohésion sociale (CUCS), etc.

2. Jean-Pierre Orfeuil (2000) fait état d'une vitesse globale moyenne en automobile de $17 \mathrm{Km} / \mathrm{h}$ pour les habitants du centre et de $23 \mathrm{Km} / \mathrm{h}$ pour ceux de banlieue, pour la mobilité locale dans les agglomérations de 300000 à 900000 habitants.

3. Un parcours plus direct par la nouvelle ligne 3 (inaugurée en avril 2012) est désormais possible.

4. Source : Horaires officiels du service d'hiver 2010-2011.

5. Ce temps, calculé par Via Michelin (http://www.viamichelin.fr/web/Itineraires), tient compte d'une partie importante du parcours sur voie rapide.

\section{RÉSUMÉS}

S'il est difficile de contester le fait que le retour récent du tramway en France est à la fois un succès politique et opérationnel, la question de la vitesse commerciale mérite d'être posée. En effet, cette dernière est calculée de terminus à terminus, ce qui masque de fortes disparités internes. En creusant davantage, on constate qu'elle peut être très faible là où il $\mathrm{y}$ a la plus forte fréquentation, et que, même si elle peut paraître suffisante, elle doit être considérée à longueur égale vis-à-vis du tracé routier le plus « évident ». Or, les lignes ont tendance à serpenter plus ou moins, ce qui allonge leur parcours par rapport à celui que suivaient les autobus antérieurement, et que suivent toujours les automobilistes puisque l'on a bien souvent évité de suivre les grands axes lorsque cela risquait d'y remettre en cause la place de l'automobile. 
Le fait de changer souvent de direction est également un handicap pour des véhicules qui ne donnent le meilleur d'eux-mêmes que lorsqu'ils peuvent rouler à vitesse nominale. Chaque courbe, qui donne lieu à une sévère limitation de vitesse, est un facteur de diminution de la vitesse commerciale. Sur certaines lignes, les vitesses réduites concernent presque $50 \%$ du parcours (c'est le cas par exemple à Reims).

Le présent article cherche à tirer les leçons de l'analyse d'un échantillon de neuf réseaux de TCSP guidés hors Île-de-France, associant tramway sur fer et modes hybrides réputés plus « souples ».

The récent come back of LRT in France is a political and an operational success, which cannot be subject to contestation. Beyond the patronage figures, it is of high interest to focus our attention to the question of the commercial speed, calcultaed from one end of the line to the other. This mode of calculation hides major internal disparities between the different parts of a specific line. Lower speed are found where the level of fréquentation is the highest. And, even if the calculated speed can be considered as sufficient, it must be put into considération with the length of the LRT route, which can be longer than the most evident bus or car route. We have noticed that most of the lines studied were more or less winding compared with the "évident " routes : an acceptable commercial speed on a longer route gives no particular advantage to LRT, and finally to public transport vs. private transport.

Frequent changes of direction also lower the performances of a LRT system. Too many curves have négative consequences on the commercial speed, because of low speed limits. On certain lines (for instance in Reims), nominal speed is available only on $50 \%$ of the total route length. This article tries to determine the reasons of the lower level of performance from a set of nine LRT networks out of the Paris Region.. This set associates single-line and more complex networks, classical tramways, LRT on tyres and « hybrid » systems (partially guided).

\section{INDEX}

Keywords : Light Rail Transit (LRT), France, lines, commercial speed, profile, performances Mots-clés : TCSP, France, lignes, vitesse commerciale, tracés, agencements, performances

\section{AUTEUR}

\section{PIERRE ZEMBR}

Université de Cergy-Pontoise - EA 4113 MRTE - 33, boulevard du Port. F-95000 CERGY-PONTOISE

(France). pierre.zembri[chez]u-cergy.fr 